\title{
Exchange-bias instability in a bilayer with an ion-beam imprinted stripe pattern of ferromagnetic/antiferromagnetic interfaces
}

\author{
Katharina Theis-Bröhl,* Maximilian Wolff, Andreas Westphalen, and Hartmut Zabel \\ Department of Physics, Ruhr-University Bochum, D-44780 Bochum, Germany \\ Jeffrey McCord \\ Leibniz Institute for Solid State and Materials Research Dresden, Institute for Metallic Materials, Helmholtzstrasse 20, \\ D-01169 Dresden, Germany
}

Volker Höink, Jan Schmalhorst, and Günther Reiss

Department of Physics, University Bielefeld, Universitätsstrasse 25, D-33615 Bielefeld, Germany

Tanja Weis, Dieter Engel, and Arno Ehresmann

Institute of Physics and Centre for Interdisciplinary Nanostructure Science and Technology (CINSaT), University of Kassel, Heinrich-Plett-Strasse 40, D-34132 Kassel, Germany

Ulrich Rücker
Institut für Festkörperforschung, Forschungszentrum Jülich GmbH, D-52425 Jülich, Germany

Boris P. Toperverg ${ }^{\dagger}$

Department of Physics, Ruhr-University Bochum, D-44780 Bochum, Germany, Institut Laue Langevin, Boîte Postale 156, Grenoble 38000, France, and Petersburg Nuclear Physics Institute, Gatchina, 188300 St. Petersburg, Russia

(Received 28 November 2005; revised manuscript received 16 March 2006; published 8 May 2006)

\begin{abstract}
We have investigated the magnetization arrangement in an in-plane stripe pattern with alternating exchangebias domains. The stripe pattern was produced by ion bombardment induced magnetic patterning, which changed locally the exchange-bias direction at the ferromagnet/antiferromagnet interface, but not the magnetic or antiferromagnetic properties of the $\mathrm{Co}_{70} \mathrm{Fe}_{30}$ and $\mathrm{Mn}_{83} \mathrm{Ir}_{17}$ layers, respectively. For the analysis of the magnetic domain structure evolution along the hysteresis loop we used a combination of experimental techniques: magneto-optical Kerr effect, Kerr microscopy, polarized neutron reflectometry, and off-specular scattering of polarized neutrons with polarization analysis. Instead of a perfect antiparallel alignment we found that the magnetization in neighboring stripes is periodically canted with respect to the stripe axis so that the net magnetization of the ferromagnetic film turns almost perpendicular to the stripes. At the same time the projection of the magnetization vector onto the stripe axis has a periodically alternating sign. The experimental observations are explained and quantitatively described within the frame of a phenomenological model, taking into account interfacial exchange bias, intralayer exchange energy, and uniaxial anisotropy. The model defines conditions which can be used for tailoring nano- and micro-patterned exchange-bias systems with different types of magnetic order.
\end{abstract}

DOI: 10.1103/PhysRevB.73.174408

\section{INTRODUCTION}

Patterned magnetic media and magnetic nanoparticles have received considerable attention recently for obtaining high package densities in magnetic recording media. ${ }^{1-3} \mathrm{Al}-$ though very high storage densities can be achieved, still several problems have to be dealt with. First, the patterned and clustered media have not yet reached high surface smoothness which is necessary for near contact recording in hard disks. Second, with decreasing feature size the superparamagnetic limit is approached. ${ }^{3}$ Third, large variations in optical constants occur which are not favorable for near-field magneto-optical technologies. ${ }^{4}$ And last but not least, long range interactions between individual magnetic elements may result in an undesirable cooperative response of nanosystems in reaction to a perturbation.
PACS number(s): 75.60.Ch, 75.60.Ej, 75.75.+a, 61.12.Ha

During the last decade, a number of techniques were developed to produce systems of nano-elements with designed magnetic properties. Among those are ion based methods, such as the ion bombardment induced magnetic patterning $(\text { IBMP })^{5-7}$ and focused ion-beam treatment. ${ }^{8-10}$ The IBMP uses ion bombardment in an external magnetic field through a resist mask to alter magnetic properties, such as anisotropy, ${ }^{11,12}$ coercivity, ${ }^{5}$ or exchange bias $(\mathrm{EB})^{5-7,13,14}$ between ferromagnetic (FM) and antiferromagnetic (AFM) layers in one part of the film, while keeping the properties of the untreated regions unchanged. With IBMP beam patterned magnetic media one avoids the problems that magnetic nanostructures and clusters face. On the other hand, interactions between the different regions in IBMP magnetic films with locally varying magnetic properties may still hamper their immediate applications. The effect of one type of such inter- 
actions, i.e., dipolar forces,${ }^{15}$ can be substantially diminished by using special geometries such as magnetic rings ${ }^{16}$ or circular magnetic disks ${ }^{17,18}$ with the magnetic flux closed within the nano-elements. However, if IBMP is used to pattern continuous films into such special geometries, the dipolar interaction is not eliminated, in contrast to the case of isolated ferromagnetic elements. ${ }^{15}$ The continuous film dictates particular conditions for the magnetic flux path through the border between neighboring structural elements. Moreover, such a patterning does not prevent the direct exchange interaction of atomic spins shared between virgin regions and those in which the magnetic properties are modified due to bombardment. The exchange coupling is usually by a few orders of magnitude stronger than the magnetic dipole-dipole interaction. However, the exchange coupling energy is proportional to the area of interfaces between structural elements in contact, while the dipolar coupling is proportional to the volume element squared. Therefore these two types of interaction can successfully compete against each other, as well as against the crystalline anisotropy, determining altogether a rather complicated magnetic cooperative behavior of the nano-system.

For a detailed study of the magnetic interactions between neighboring magnetic elements in a continuous film we designed a model system. Considering that the model system should be of technological interest on the one hand, and should allow the application of several different and efficient experimental techniques for complementary analysis on the other hand, such as polarized neutron reflectivity (PNR), longitudinal and transverse Kerr magnetometry (MOKE), and Kerr microscopy (KM), we have chosen a film with imprinted in-plane stripelike magnetic domains. The stripes are expected to display alternating magnetization directions in the remanent demagnetized state. To realize an antiparallel alignment of neighboring stripes, we have taken advantage of the unidirectional anisotropy of the exchange-bias (EB) systems, modified by IBMP.

A subsequent detailed analysis of the PNR and MOKE data has revealed that the magnetization in neighboring stripes is not aligned antiparallel to each other, as expected, but is appreciably tilted away from the stripe symmetry axes of the system. This means that the interstripe interaction generates an instability of the system with respect to a spontaneous appearance of the magnetization directed perpendicular to the stripes. Simple theoretical considerations show that due to the competition between exchange coupling within the FM layer and alternating interfacial EB field from the AFM sublayers, the expected antiparallel alignment is, indeed, unstable with respect to ferromagnetic ordering. This FM order coexists with AFM order over a wide range of exchange coupling parameters.

This paper is organized as follows: We first describe in Sec. II the methods for producing magnetic microstripes with alternating direction of EB essentially without surface topography differences and the experimental techniques used for analyzing their magnetization reversal. In Sec. III we present the experimental results obtained with the different methods, and in Sec. IV we discuss the experimental results on the basis of theoretical simulations of our experimental neutron data. In Sec. V we summarize our findings and provide a theoretical explanation for the spontaneous breaking of the symmetry.

\section{EXPERIMENTAL DETAILS}

\section{A. Sample preparation}

The sample studied in this paper is an exchange-biased FM/AFM bilayer $\mathrm{Ta}(5 \mathrm{~nm}) / \mathrm{Co}_{70} \mathrm{Fe}_{30}(30 \mathrm{~nm}) / \mathrm{Mn}_{83} \operatorname{Ir}_{17}(15$ $\mathrm{nm}) / \mathrm{Cu}(30 \mathrm{~nm}) / \mathrm{SiO}_{2}(50 \mathrm{~nm}) / \mathrm{Si}(111)$ (the given thicknesses are nominal values). $\mathrm{Co}_{70} \mathrm{Fe}_{30}$ was chosen because of its highest spin polarization among the CoFe alloys. The film structure was prepared by magnetron sputtering with a base pressure below $1 \times 10^{-7} \mathrm{mbar}$ at an $\mathrm{Ar}$ pressure of 3 $\times 10^{-3}$ mbar. The initial EB direction was set by field cooling in a magnetic in-plane field of $H_{\text {f.c. }}=1 \mathrm{kOe}$ after an annealing step for $1 \mathrm{~h}$ at $275{ }^{\circ} \mathrm{C}$ which is above the blocking temperature of the antiferromagnetic material. A loop shift of $H_{\mathrm{EB}, 0}=35$ Oe together with a coercive field $H_{\mathrm{c}, 0}=15 \mathrm{Oe}$ is obtained after thermal treatment. Subsequently the film was covered by a photoresist, which was patterned into equally spaced stripes with a width of $d=2.5 \mu \mathrm{m}$ and a periodicity of $\Lambda=5 \mu \mathrm{m}$. Then the sample was bombarded with $\mathrm{He}^{+}$ions with a fluency of $1 \times 10^{15}$ ions $/ \mathrm{cm}^{2}$ at $10 \mathrm{keV}$. The resist thickness of $0.5 \mu \mathrm{m}$ is sufficient to protect the covered regions from the $\mathrm{He}^{+}$exposure. During bombardment a magnetic field of $H_{\text {ion }}=1 \mathrm{kOe}$ aligned opposite to the initial EB direction was applied. This resulted in a stripelike pattern with alternate sign of the unidirectional anisotropy, and hence the sign of the EB in neighboring stripes. ${ }^{5}$ The effect of ion bombardment using $\mathrm{He}^{+}$ions on exchange biased systems was studied in detail by one of the coauthors (A.E.). ${ }^{13}$ Two possible effects are discussed in this work: hyperthermal local heating and defect creation in the AFM grains (causing enhancement of $H_{\mathrm{EB}}$ ) and defect formation at the FM/AFM interface (causing a reduction of $H_{\mathrm{EB}}$ ). Using x-ray absorption and magnetic circular dichroism Schmalhorst et $a l$. found that the distribution of Co and Mn within the film is not significantly altered by using a moderate ion dose. ${ }^{19}$

In Secs. II B and II C some general remarks on the experimental techniques of vector-MOKE and PNR are made, which may be skipped in case of familiarity with these methods.

\section{B. Magneto-optical Kerr effect}

We have analyzed the magnetization reversal with a vector-MOKE set up as described in Ref. 20. The HeNe-laser was polarized parallel to the sample plane and perpendicular to the reflection plane. Vector-MOKE allows one to record the in-plane projection of the magnetization vector parallel and perpendicular to the reflection plane. First the Kerr angle $\theta_{\mathrm{K}}$ is measured in the standard longitudinal configuration. In our case the magnetic stripes were aligned parallel to the reflection plane and parallel to the external magnetic field direction. Then $\theta_{\mathrm{K}}=\theta_{\mathrm{K}}^{\mathrm{L}}$ measures solely the "longitudinal" magnetization projection $\theta_{\mathrm{K}}^{\mathrm{L}} \propto M_{\mathrm{K}}^{\mathrm{L}}=M \cos \gamma$ onto the intersection between the reflection plane and the sample surface, where $\gamma$ denotes the tilt angle of the magnetization with respect to the external field. The latter was cycled parallel to 
the exchange bias axis, which does not mean necessarily, as we shall see, that $\gamma=0$. In order to determine the angle $\gamma$ $\neq 0$ unambiguously, one needs additional measurements. Indeed, the Kerr angle is proportional to the product of two factors, e.g., $\cos \gamma$ and magnetization $M=|\boldsymbol{M}|$, and in general, due to domains, $M \leqslant M_{s}\left(M_{s}\right.$ is the magnetization in saturation). In order to determine both, $\gamma$ and $M$, we have measured the hysteresis again after rotating the sample and the magnetic field direction simultaneously by $90^{\circ}$ about the normal to the surface, such that the magnetic field maintains its orientation with respect to the bias axis. The Kerr rotation $\theta_{\mathrm{K}}$ is still measured in the longitudinal configuration, but this time we measure the "transverse" magnetization projection $\theta_{\mathrm{K}}=\theta_{\mathrm{K}}^{\mathrm{T}} \propto M_{\mathrm{K}}^{\mathrm{T}}=M \sin \gamma$.

Assuming that $\theta_{\mathrm{K}}$ and $M_{\mathrm{K}}$ are linearly related and that the linear proportionality coefficients between $\theta_{\mathrm{K}}^{\mathrm{L}}$ and $M_{\mathrm{K}}^{\mathrm{L}}$ and between $\theta_{\mathrm{K}}^{\mathrm{T}}$ and $M_{\mathrm{K}}^{\mathrm{T}}$ are equal, one finds

$$
\tan \gamma=\frac{\theta_{\mathrm{K}}^{\mathrm{T}}}{\theta_{\mathrm{K}}^{\mathrm{L}}}=\frac{M_{\mathrm{K}}^{\mathrm{T}}}{M_{\mathrm{K}}^{\mathrm{L}}},
$$

which determines the tilt angle $\gamma$.

In saturation, one expects that $\gamma=0$ and $M_{s} \propto \theta_{\mathrm{K}}^{\mathrm{s}}$, where $\theta_{\mathrm{K}}^{\mathrm{s}}$ is the Kerr angle measured in saturation. Furthermore, the length of the magnetization vector $M=|\boldsymbol{M}|=\sqrt{M_{\mathrm{L}}^{2}+M_{\mathrm{T}}^{2}}$ is determined as

$$
M=M_{s} \frac{\sqrt{\left(\theta_{K}^{L}\right)^{2}+\left(\theta_{K}^{T}\right)^{2}}}{\theta_{K}^{\mathrm{s}}} .
$$

Equations (1) and (2) allow us to completely determine the magnetization vector $\boldsymbol{M}$ averaged over the spot illuminated by the laser beam.

Further insight into the microscopic rearrangement of magnetization during cycling with an external field along the exchange bias axis was achieved by a high resolution magneto-optical Kerr microscope $(\mathrm{KM})^{21}$ that is sensitive to directions orthogonal to the field. ${ }^{22,23}$ The KM images provide good input data for constructing a remagnetization model which was then used to refine the physical parameters required for fitting polarized neutron reflectometry (PNR) measurements.

\section{Polarized neutron reflectometry}

Neutron scattering experiments were carried out with the ADAM reflectometer at the Institut Laue-Langevin, Grenoble, France, ${ }^{24}$ and with the HADAS reflectometer at the FRJ-2 reactor in Jülich, Germany. The measurements at ADAM and HADAS were performed with fixed neutron wavelengths of $\lambda=0.441$ and $0.452 \mathrm{~nm}$, respectively, in both cases from a cold neutron source. The HADAS reflectometer is equipped with a polarization analyzer covering the whole area of the position sensitive detector, allowing one to measure efficiently off-specular scattering with full polarization analysis. We performed PNR experiments using a scattering geometry as shown in Fig. 1. A magnetic field was applied along the $y$-axis aligned parallel to the microstripes and perpendicular to the scattering plane. The $z$-axis is chosen along the normal to the sample, which is also the direction of the

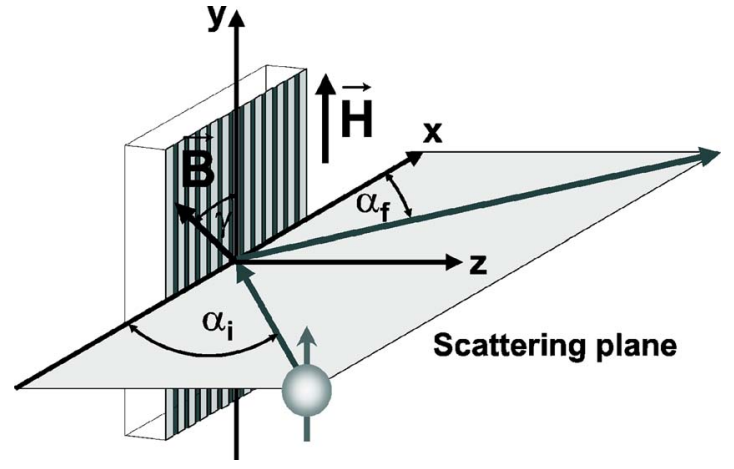

FIG. 1. (Color online) Sketch of the neutron scattering geometry. The magnetic field $\boldsymbol{H}$ is applied perpendicular to the scattering plane. $\alpha_{i}$ and $\alpha_{f}$ refer to the incident and exit angles of the neutrons with respect to the sample surface.

scattering vector for specular reflectivity. The $x$-axis lies within the scattering plane perpendicular to the stripes.

The magnetic induction vector $\boldsymbol{B}=\boldsymbol{H}+4 \pi \boldsymbol{M}$ (which is basically due to the magnetization vector) is assumed to lie in the sample plane (in-plane shape anisotropy). Furthermore, $\boldsymbol{B}$ may have an angle $\gamma$ with respect to the external magnetic field $\boldsymbol{H}$ applied along the $y$-axis. We assume that a monochromatic neutron beam incident onto the sample surface under the angle $\alpha_{i}$ is scattered under the glancing angle $\alpha_{f}$, so that for specular reflection $\alpha_{i}=\alpha_{f}$. The incident polarization vector is directed either parallel or antiparallel to $\boldsymbol{H}$ and perpendicular to the scattering plane.

Specular PNR provides information similar but not identical to vector-MOKE. Indeed, neutrons are reflected specularly from the mean optical potential $U_{N}+U_{M}$ averaged over the coherence range. In case of coherent neutron scattering, the nuclear part $U_{N}$ does not affect the neutron spin states, while the interaction potential $U_{M}$ of neutron spins with the mean magnetic induction $\boldsymbol{B}$ splits the spin component of the neutron wave inside the magnetized layer. As a result, the doublet of reflection amplitudes has two components $R_{ \pm}$corresponding to the reflection of positive, or, respectively, negative neutron spin projections onto the direction of $\boldsymbol{B}$, and the equation for reflectivity $\mathcal{R}\left(P_{i}, P_{f}\right)$ reads: ${ }^{.5,26}$

$$
\begin{aligned}
\mathcal{R}\left(P_{i}, P_{f}\right)= & \frac{1}{4}\left\{\left(\left|R_{+}\right|^{2}+\left|R_{-}\right|^{2}\right)\left(1+P_{i} P_{f}\right)+\left(\left|R_{+}\right|^{2}-\left|R_{-}\right|^{2}\right)\right. \\
& \left.\times\left(P_{i}+P_{f}\right) \cos \gamma-\left|R_{+}-R_{-}\right|^{2} P_{i} P_{f} \sin ^{2} \gamma\right\},
\end{aligned}
$$

where $P_{i}= \pm\left|P_{i}\right|$ and $\left|P_{i}\right| \leqslant 1$ is the efficiency of the polarizing device, while $P_{f}= \pm\left|P_{f}\right|$ and $\left|P_{f}\right| \leqslant 1$ is the efficiency of the polarization analysis. The flippers before and after the sample alternate signs of the incident or outgoing neutron polarization, respectively, so that two non-spin-flip (NSF), $\mathcal{R}^{ \pm \pm}=\mathcal{R}\left( \pm P_{i}, \pm P_{f}\right)$, and two spin-flip (SF), $\mathcal{R}^{ \pm \mp+}$ $=\mathcal{R}\left( \pm P_{i}, \mp P_{f}\right)$, reflection coefficients are measured.

If in an ideal case (i.e., $\left|P_{i}\right|=\left|P_{f}\right|=1$ ) neutrons are polarized collinearly with $\boldsymbol{B}$, then there are only two non-spin-flip (NSF) reflection coefficients: $\mathcal{R}^{++}=\left|R_{+}\right|^{2}$ and $\mathcal{R}^{--}=\left|R_{-}\right|^{2}$, corresponding to reflection of neutrons polarized parallel and antiparallel to $\boldsymbol{B}$, respectively. In this case reflection does not 
change the spin states, i.e., the spin-flip (SF) reflection coefficients $\mathcal{R}^{+-}=\mathcal{R}^{-+}=0$. The spin asymmetry (SA) $\mathcal{R}^{++}-\mathcal{R}^{--}$ gives access to the mean magnetization averaged over the neutron coherence range. It should be kept in mind that the amplitudes $R_{ \pm}$are complex nonlinear functions of the mean magnetization, which in contrast to vector-MOKE, can be determined only as a parameter via fitting the data to a theoretical model. This complication of PNR is, on the other hand, compensated by the possibility to obtain absolute values for the mean magnetization without additional calibration: the nuclear and magnetic scattering length densities are well-tabulated for a vast majority of chemical elements, compounds, and isotopes.

There are two possibilities for $R_{+}=R_{-}$and a vanishing SA: either the mean magnetization is zero for some reasons in the case that the magnetic domains are smaller than the neutron coherence range, or the mean magnetization is directed perpendicularly to the polarization. If the polarization is not collinear with the mean magnetization then $\mathcal{R}^{+-}$ $=\mathcal{R}^{-+} \neq 0$, and these $\mathrm{SF}$ reflectivities reach maximum values if the mean magnetization is oriented perpendicular to the polarization analysis axis. In general, SF reflectivities are proportional ${ }^{37}$ to the mean square of the magnetization component perpendicular to the polarization axis, i.e., to $\left\langle\sin ^{2} \gamma\right\rangle$. Technical difficulties hamper an application of the same trick as used in vector-MOKE in order to probe both $\langle\sin \gamma\rangle$ and $\langle\cos \gamma\rangle$ and to determine the direction and the modulus of the mean magnetization. Instead, one has access only to $\langle\cos \gamma\rangle$ which can be determined from NSF reflectivities and to $\left\langle\sin ^{2} \gamma\right\rangle$ deduced from the evaluation of the SF signal at the general constraint $\Delta=\left\langle\sin ^{2} \gamma\right\rangle-\langle\sin \gamma\rangle^{2} \geqslant 0$.

However, the latter very important quantity is inaccessible for vector-MOKE, which coherently averages magnetic fluctuations over the laser spot illuminating the surface. Its area is typically a fraction of $\mathrm{mm}^{2}$. The neutron source, on the contrary, is essentially incoherent, and the coherency range of the beam is determined by its collimation and monochromatization. The neutron beam is well-collimated in the reflection plane $(x-z)$, while the collimation is usually relaxed along the $y$-axis. Hence the coherence length parallel to the stripes amounts to only about $10 \mathrm{~nm}$, whereas in the perpendicular direction parallel to the $x$-axis it is rather extended at shallow angles of incidence. This is due to the fact that the uncertainty in the $x$-coordinate is proportional to $\lambda /(\alpha \Delta \alpha)$, where $\alpha \sim \alpha_{i} \sim \alpha_{f} \ll 1$, and $\Delta \alpha$ accounts for the uncertainties in $\alpha_{i}$ and $\alpha_{f}$ due to collimation and detector resolution. As a result, the coherence length projected along the $x$-axis may be enhanced up to a fraction of a millimeter, i.e., up to a scale which is comparable to that probed by MOKE. Due to the strong asymmetry in the coherency properties, a measured signal is the result of an incoherent average along the $y$-direction and a coherent reflection from the mean optical potential along the $x$-axis. This enables one to measure coherent effects along the $x$-direction, such as off-specular Bragg diffraction from a periodic structure. In the present case a periodic alteration in the local magnetization is produced by exchange bias patterning. Due to variations in the tilt angles $\gamma=\bar{\gamma}+\Delta \gamma$ against the applied field, one may observe local periodic deviations $\Delta \gamma$ with respect to the mean
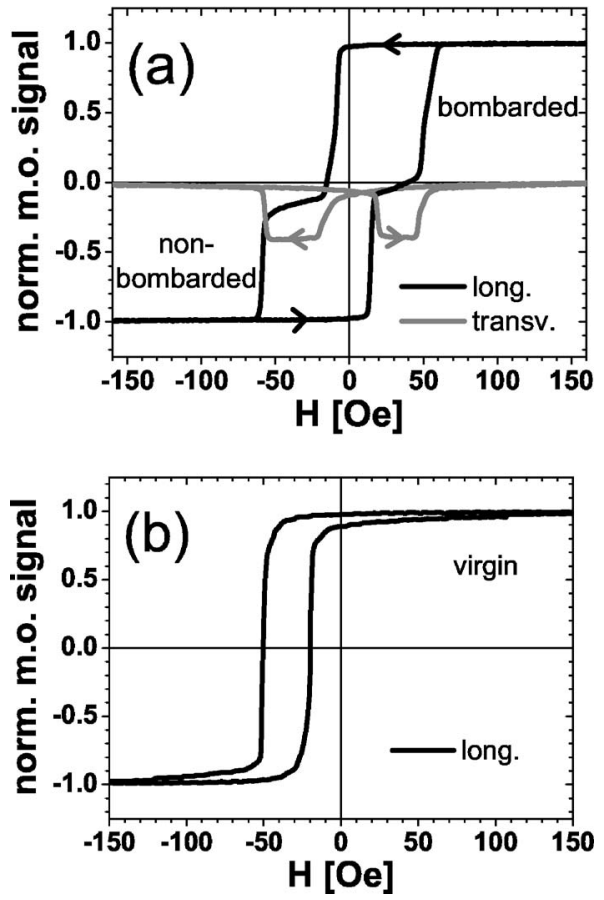

FIG. 2. (a) Vector-MOKE hysteresis loops of a sample magnetically patterned in stripes by IBMP and measured with the field applied along the EB axis and showing the longitudinal and transverse components of the magnetization vector. The longitudinal loop corresponds to $H_{\mathrm{EB} \text {,nonbom }}=22.5 \mathrm{Oe}$ and $H_{\mathrm{EB} \text {,bom }}=-21.5 \mathrm{Oe}$, and $H_{\mathrm{c}, \text { nonbom }}=36.5 \mathrm{Oe}$ and $H_{\mathrm{c}, \mathrm{bom}}=30.5 \mathrm{Oe}$ for the nonbombarded and the bombarded part, respectively. The reversal of the differently treated areas occur at $H_{\mathrm{c} 1 \text {,asc }}=14 \mathrm{Oe}, H_{\mathrm{c} 2 \text {, asc }}=52$ Oe for the ascending branch and at $H_{\mathrm{c} 1 \text {,desc }}=-9 \mathrm{Oe}, H_{\mathrm{c} 2 \text {, desc }}=-59$ Oe for the descending branch. (b) MOKE hysteresis loop performed at the virgin sample and showing the longitudinal component of the magnetization vector. The loop corresponds to $H_{\mathrm{EB}, 0}=35 \mathrm{Oe}$ and $H_{\mathrm{c}, 0}$ $=15 \mathrm{Oe}$.

value $\bar{\gamma}$. Furthermore, deviations from a perfect periodicity can be quantified via evaluation of diffuse off-specular scattering observed either around Bragg diffraction of zeroth order or around Bragg reflections of higher order.

\section{EXPERIMENTAL RESULTS}

\section{A. MOKE magnetometry}

We performed the MOKE measurements with the field along the EB axis which is parallel to the IBMP magnetically patterned stripe edges. Vector-MOKE results are displayed in Fig. 2(a), and in Fig. 2(b) they are compared with results from the virgin state of the sample. In Fig. 2(a), a two step magnetization reversal is clearly visible in the longitudinal MOKE hysteresis, corresponding to a successive remagnetization of two regions with alternative directions of the exchange bias field. Both the magnetic loop shift and the coercivities are comparable for descending and ascending branches. The effective loop shift $H_{\mathrm{EB}, \text { nonbom }}=22.5 \mathrm{Oe}$ of the nonbombarded regions is reduced relative to $H_{\mathrm{EB}, 0}=35 \mathrm{Oe}$ determined for the virgin state of the sample [Fig. 2(b)]. Note that the plateau in the descending loop branch in Fig. 2(a) 


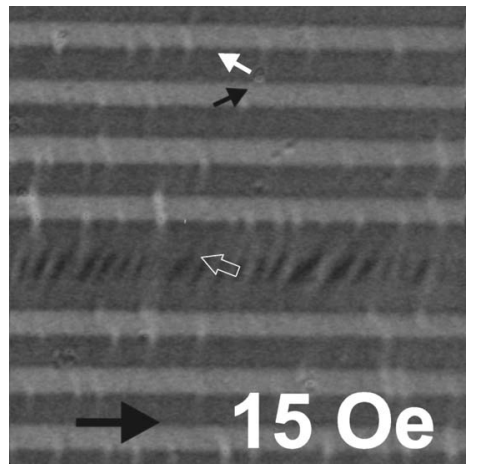

FIG. 3. Kerr microscopy image taken with the field along the EB axis. The image was recorded with a mixed Kerr sensitivity tuned nearly to the transverse magnetization direction. The magnetic field was 15 Oe.

occurs not exactly at zero magnetization. Furthermore, the slope of the magnetization curve corresponding to the reversal of magnetization in the bombarded regions is smaller compared to that of the nonbombarded ones. This may be due to defects created by the ions. Otherwise, the general behavior of the ascending (nonbombarded microstripes reverse first) and descending branches of the loop (bombarded microstripes reverse first) are quite similar.

The transverse hysteresis loop in Fig. 2(a) shows, however, a remarkable and rather unusual feature: a considerable transverse component of magnetization with plateaus in the field range where we expect an antiparallel alignment of the magnetization in bombarded and nonbombarded parts of the sample. Another striking feature is that both plateaus appear in the negative half of the diagram indicating that the transverse component of the magnetization has one and the same orientation for both loop branches.

\section{B. Kerr microscopy}

A detailed KM analysis of the present sample was recently published. ${ }^{22}$ With the microscope tuned to longitudinal magnetic contrast it was shown that starting from saturation magnetization the reversal proceeds through an antiparallel alignment of magnetization by head-on domain wall propagation along the stripes. In addition, the transverse magnetization contrast provides a rather detailed insight into the microscopic arrangement at different stages of the remagnetization process.

A representative example of a $\mathrm{KM}$ image taken with the Kerr sensitivity set close, but not exactly, to the transverse direction, is reproduced in Fig. 3. This mode was chosen to show all important features discussed below. The image was taken in the ascending loop branch at a field just above the first reversal $H_{\mathrm{c} 1 \text {,asc }}$. At this field, the magnetization in the bombarded parts of the sample (corresponding to the darkgray MOKE contrast) is not yet reversed, while it is reversed in all but one nonbombarded stripes (light-gray MOKE contrast). The nonbombarded stripe in the middle of the picture where the magnetization is not yet reversed exhibits typical features of a reversal taking place through ripple domains connected to an incoherent magnetization rotation. Note that rippling is typical for Co and Co rich CoFe alloys, ${ }^{15,28}$ and in our sample always occurs around both coercive fields $H_{\mathrm{c} 1}$ and $H_{\mathrm{c} 2}$. In the antiparallel state, the magnetization between the stripes is separated by Néel-wall-like structures (dark MOKE contrast between the stripes) with a preferred sense of magnetization rotation. In Fig. 3 it is also apparent that stripes with antiparallel magnetization direction are not uniformly magnetized. They rather consist of areas providing a gray MOKE contrast, interspersed by small transverse domains seen with bright MOKE contrast indicative of an opposite rotational direction. With KM we did not detect a significant difference between reversals via descending or ascending branches when the field was applied along the EB direction.

As mentioned before, the image in Fig. 3 was obtained with a KM mode preferentially sensitive to the transverse magnetization component, i.e., perpendicular to the stripes. Therefore, the image may give the impression that the transverse components are dramatically different in neighboring stripes. This is not the case, as can be seen from Kerr microscopy images with an extremely accurate setting of the Kerr sensitivity. In Fig. 4 we demonstrate two representative examples of KM images, one with pure longitudinal (left picture) and one with pure transverse sensitivity (right picture), respectively. The images were taken in the ascending loop branch at a field between $H_{\mathrm{c} 1 \text {,asc }}$ and $H_{\mathrm{c} 2 \text {,asc }}$, the first and the second reversal. At this field the magnetization in bombarded parts of the sample (corresponding to the dark-gray MOKE contrast in the left image) is not yet reversed, while it is completely reversed in the nonbombarded parts (light-gray MOKE contrast in the left-hand image). The image with longitudinal sensitivity shows a very strong contrast between neighboring stripes, corresponding to different longitudinal components of the magnetization (parallel and antiparallel to the applied field). Note that this contrast is by far stronger than that in Fig. 3. Both types of stripes show, on the con-
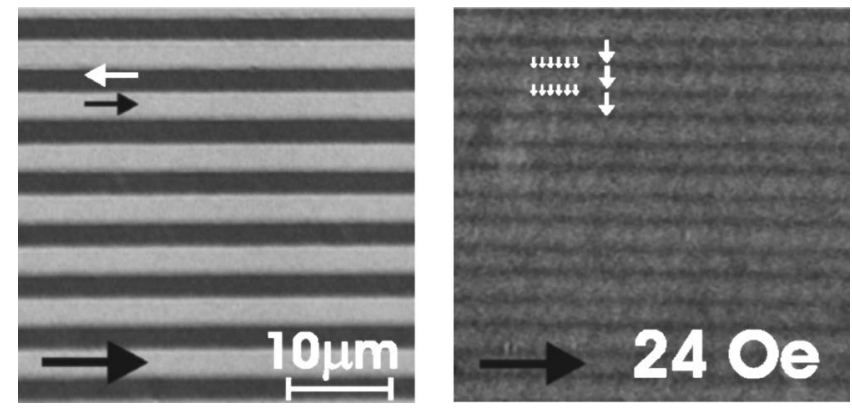

FIG. 4. Kerr microscopy images taken with the field along the EB axis. The image on the left side was recorded with the KM mode sensitive to the longitudinal magnetization components. The image on the right side was taken with the KM mode precisely set to be sensitive to variations of magnetization projections perpendicular to the stripes. The magnetic field was 24 Oe ascending from negative saturation. The medium size black and white arrows show the respective magnetization component within the stripes (longitudinal component in the left and transverse component in the right image). The small size arrows indicate the transverse magnetization in the walls, hardly visible between stripes, and the large size arrows point to the orientation of the applied field. 


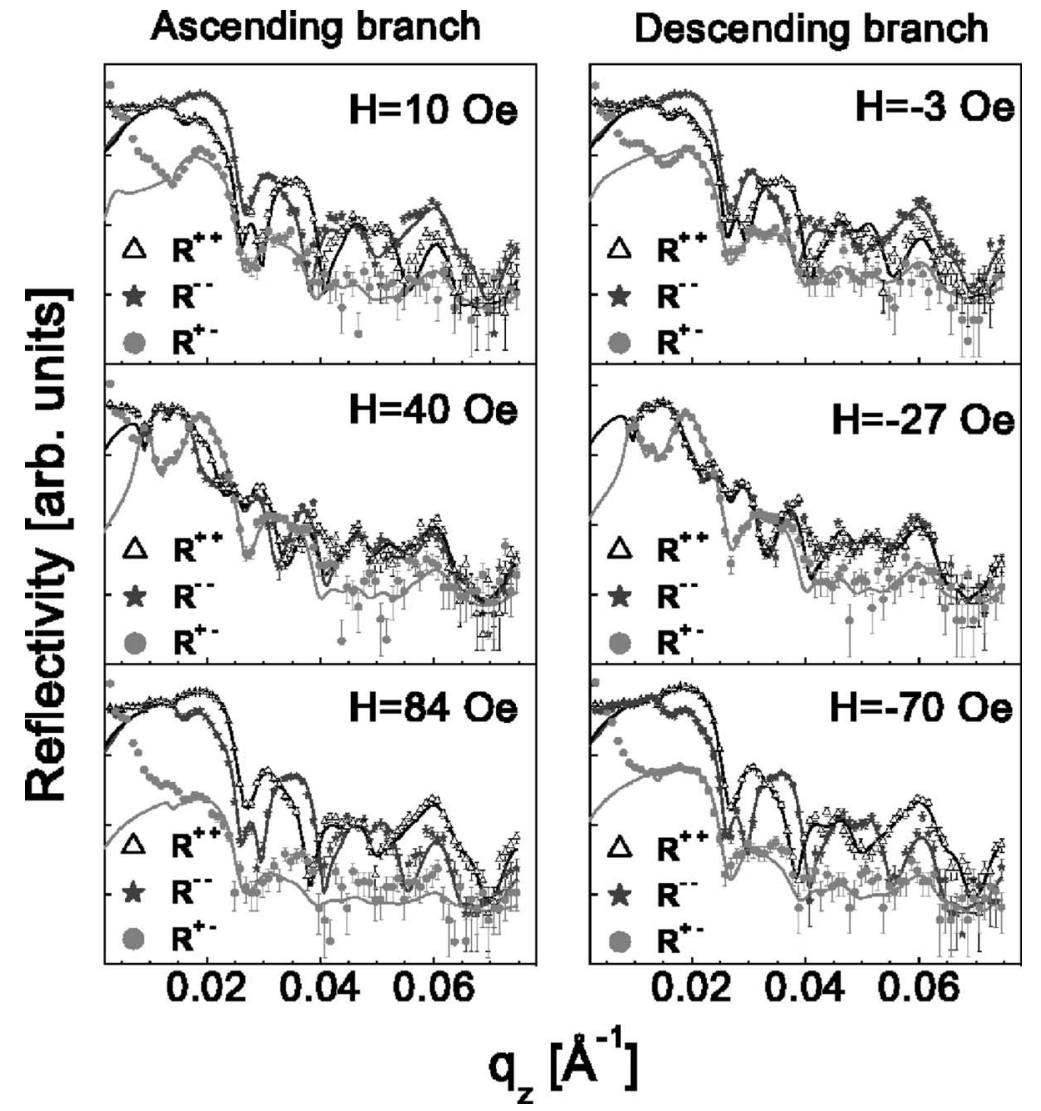

FIG. 5. Polarized neutron reflectivity measurements performed at different applied magnetic field. The symbols present measurements of non-spin-flip reflectivities $\mathcal{R}^{++}$and $\mathcal{R}^{--}$and one spin-flip reflectivity $\mathcal{R}^{+-}$. The lines represent fits to the data points. The sign of the field value corresponds to that in MOKE measurements in Fig. 2. trary, the same gray MOKE scale with practically no contrast in the image with transverse Kerr sensitivity. This observation provides a strong evidence for the continuity of the transverse magnetization components across the border between neighboring stripes in our sample at fields between $H_{\mathrm{c} 1 \text {,asc }}$ and $H_{\mathrm{c} 2 \text {,asc }}$. The stripes are separated by hardly visible lines with a dark MOKE contrast. Such lines are attributed to the Néel-wall-like structures ${ }^{38}$ formed in the antiparallel state. ${ }^{22}$

The observation that the transverse components are not much different in neighboring stripes is in agreement with Maxwell's equations which request a continuity of the projection of magnetic induction onto the normal to the interface between two different magnetic media. Such interfaces are naturally formed along the border between bombarded and nonbombarded stripes. The longitudinal magnetization (and magnetic inductance) component, on the contrary, can easily vary from stripe to stripe as well as along the stripes. In the latter case they form domain walls across the stripes.

\section{Specular neutron reflectivity}

Specular polarized neutron reflectivity (PNR) was measured using a pencil-like as well as a position sensitive detector (PSD). The PSD records, additionally to the specular reflection from the mean neutron optical potential, magnetic Bragg diffraction from the periodic stripe array and offspecular diffuse scattering from domains smaller than the coherence range.

The data were taken at several field values of the ascending and descending branches of the hysteresis loop. In order to avoid neutron depolarization, the magnetic field at the sample position was always kept parallel to the field guiding the neutron polarization. Prior to each measurement in an ascending field, the sample was saturated in a negative field. In order to take data corresponding to negative fields, the sample was rotated by $180^{\circ}$. In Fig. 5, several representative specular reflectivity curves measured at the ADAM reflectometer for both loop branches are displayed. The scans in the top row show measurements taken at fields just below the first reversal at $H_{\mathrm{c} 1}$, the data of the scans in the middle row are taken in the antiparallel state between $H_{\mathrm{c} 1}$ and $H_{\mathrm{c} 2}$, and the data in the bottom row are taken above $H_{\mathrm{c} 2}$, at almost saturation.

The non-spin-flip (NSF) reflectivity curves in the first row look quite similar to those in the last one, if the (NSF) reflectivity $\mathcal{R}^{++}$in the latter row is substituted by its NSF counterpart $\mathcal{R}^{--}$, and vice versa. This symmetry is just due to the opposite magnetization state of the sample in the upper and lower rows. The spin-flip (SF) reflectivity $\mathcal{R}^{+-}$in the first row, however, is bigger compared to that measured in saturation, where it is close to the limit determined by the efficiency of the polarization devices. This difference can be explained by the KM images shown in Ref. 22 and in Fig. 3. Around $H_{\mathrm{c} 1}$, a number of ripple domains develop. Rippling may lead to an increased SF reflectivity in the case of a cooperative behavior of the stripe magnetization as was observed in our previous study ${ }^{15}$ carried out at lithographically patterned $\mathrm{Co}_{70} \mathrm{Fe}_{30}$ microstripes. In any case, ripple domains manifest themselves via strong SF off-specular diffuse scattering also recorded in our present experiment. 
TABLE I. Results of the fits to the polarized neutron reflectivity data. NSLD and MSLD refer to nuclear scattering length density and magnetic scattering length density, respectively.

\begin{tabular}{cccc}
\hline \hline Layer & Thickness & NSLD & MSLD \\
\hline $\mathrm{Co}_{70} \mathrm{Fe}_{30}$ & $(26.7 \pm 0.4) \mathrm{nm}$ & $3.75 \times 10^{-6} \AA^{-6}$ & $4.93 \times 10^{-6} \AA^{-6}$ \\
Intermixed & $(1.3 \pm 0.5) \mathrm{nm}$ & $2.43 \times 10^{-6} \AA^{-6}$ & $4.34 \times 10^{-6} \AA^{-6}$ \\
$\mathrm{Mn}_{83} \mathrm{Ir}_{17}$ & $(15.2 \pm 0.1) \mathrm{nm}$ & $-0.78 \times 10^{-6} \AA^{-6}$ & 0 \\
\hline \hline
\end{tabular}

For measurements taken at field values between $H_{\mathrm{c} 1}$ and $H_{\mathrm{c} 2}$ (middle row of Fig. 5), the NSF reflectivities are almost degenerate. The spin asymmetry is $S A \approx 0$, indicating that the mean magnetization averaged over the neutron coherence range has almost no projection onto the direction of the applied field. This agrees with observations from the longitudinal hysteresis loop in Fig. 2 for the step region. This result is also expected in the case of an antiparallel alignment of the magnetization vector in neighboring stripes. At the same field values, however, we observe a very strong SF signal comparable to the NSF intensities. This is in agreement with a large magnetization component perpendicular to the applied field as already noted at transverse MOKE and KM.

We fitted the specular PNR data using an originally developed least-squares software package, ${ }^{27}$ which allows simultaneous evaluation of all four measured reflectivities in one cycle. It is based on the superiterative algorithm, ${ }^{29}$ which generalizes the Parratt ${ }^{30}$ routine for neutron reflectivity from a sequence of magnetic layers at arbitrary orientations between layer magnetization vectors, and for different incoming and outgoing polarizations. As starting parameters of our fits, we used the film thicknesses and the nuclear and magnetic scattering lengths densities as obtained from fits to PNR measurements from the virgin sample. ${ }^{27}$

Fine-tuning of film thicknesses was achieved by fits of the data in the saturated state. The corrected values of our layer stack are $\mathrm{TaO}-\mathrm{Ta}(8.7 \mathrm{~nm}) / \mathrm{Co}_{70} \mathrm{Fe}_{30}(28.0 \mathrm{~nm}) /$ $\mathrm{Mn}_{83} \operatorname{Ir}_{17}(15.2 \mathrm{~nm}) / \mathrm{Cu}(28.4 \mathrm{~nm}) / \mathrm{SiO}_{2}(50.5 \mathrm{~nm}) / \mathrm{Si}(111)$ which are very close to the nominal ones. Some of the nuclear and magnetic scattering length densities were also released in the fit and acquired noticeable deviations from the values of the untreated sample, in particular for CoFe. This may be attributed to the influence of ion bombardment which, as expected, affects mostly the region close to the FM/AFM interface. In order to account for this effect, an interlayer between $\mathrm{CoFe}$ and MnIr was assumed to further refine the fitting routine. The results of our fits are listed in Table I including the interlayer with a thickness of $1.3 \mathrm{~nm}$ and with nuclear and magnetic scattering length densities in-between those of CoFe and MnIr.

We now fixed the field independent parameters, e.g., layer thicknesses and scattering length densities obtained from the fit at saturation, and used them as input for the fits to the field dependent data. The complete set of reflectivities, $\mathcal{R}^{++}, \mathcal{R}^{--}$, $\mathcal{R}^{+-}$, and $\mathcal{R}^{-+}$, was then fitted by varying only three field dependent parameters, $\langle\cos \bar{\gamma}\rangle,\left\langle\sin ^{2} \bar{\gamma}\right\rangle$, and $\langle\cos \Delta \gamma\rangle_{\text {coh }}$, under the constraint $\langle\cos \bar{\gamma}\rangle^{2} \leqslant\left\langle\cos ^{2} \bar{\gamma}\right\rangle=1-\left\langle\sin ^{2} \bar{\gamma}\right\rangle$. The averaging goes in two steps. The first one accounts for fluctuations in the local magnetization direction on a scale smaller than the coherence range, and requiring a coherent addition of amplitudes. The second one runs over the total illuminated area, and requires the incoherent addition of scattering cross sections. Fluctuations in angles around the direction of the mean magnetization on a scale smaller than the neutron coherence range are denoted as $\Delta \gamma$. The average direction is determined by the angle $\bar{\gamma}$ due to the equation $\langle\sin \Delta \gamma\rangle_{\mathrm{coh}}$ $=0$ (the average over transverse fluctuations within the coherence range is cancelled out). The neutron coherence range is much smaller than the sample area, but still comprises a certain number of stripes and small domains. For our particular case, $\Delta \gamma$ accounts for periodic deviations of the magnetization directions in neighboring stripes responsible for Bragg diffraction. This is discussed in the next section, while for now we just mention that $\Delta \gamma$ reduces the magnetic part of the mean optical potential and thus affects the reflection amplitudes $R_{ \pm}$in Eq. (3).

Due to the fact that the neutron coherence area covers only a very small fraction of the sample surface, the magnetization averaged over this area may vary along the sample surface depending on a particular point (defined with uncertainty of the order of the coherence range). Then the observed reflectivity is proportional to the incoherent sum of intensities reflected from different coherence areas, which form narrow bars covering the total sample area. The regions in which the magnetization has a component parallel to the external field contribute to a difference between the two NSF reflectivities. The regions in which the magnetization has a component perpendicular to the external field cause SF reflection with an intensity proportional to $\sin ^{2} \gamma$. All this was accounted for in the second step of incoherent averaging over the sample surface: $\langle\cos \gamma\rangle=\langle\cos \bar{\gamma}\rangle\langle\cos \Delta \gamma\rangle_{\text {coh }}$, and substitution of $\cos \gamma$ by $\langle\cos \bar{\gamma}\rangle$ and $\sin ^{2} \gamma$ by $\left\langle\sin ^{2} \bar{\gamma}\right\rangle$ in Eq. (3) provided that $R_{ \pm}$depends on the reduced magnetic optical potential $\left\langle U_{M}\right\rangle_{\mathrm{coh}} \propto 4 \pi M_{S}\langle\cos \Delta \gamma\rangle_{\mathrm{coh}}$.

\section{Bragg diffraction and off-specular scattering}

Figure 6 displays some of the experimental data taken with the HADAS reflectometer as a function of the angle of incidence, $\alpha_{i}$, and angle of scattering, $\alpha_{f}$, collected into intensity maps. We measured maps of all four scattering cross sections $I^{++}, I^{--}, I^{+-}$, and $I^{-+}$, but we present only the results for $I^{++}$and $I^{+-}$. The specular reflection ridge runs along the diagonal, where $\alpha_{i}=\alpha_{f}$. For the specular ridge it holds: $I^{++}$ $\propto \mathcal{R}^{++}$and $I^{+-} \propto \mathcal{R}^{+-}$. At $\alpha_{i} \neq \alpha_{f}$ the scattering maps exhibit two remarkable features. The first one is the intensity of Bragg diffraction concentrated along curved lines $\cos \alpha_{i}$ $-\cos \alpha_{f} \approx n(\lambda / \Lambda)$, where $n$ denotes the order of diffraction and $\Lambda=5 \mu \mathrm{m}$ is the period. Bragg scattering is clearly apparent in the first two columns of maps in Fig. 6. It occurs due to the periodic variation of the magnetization across the striped pattern and, in particular, due to the periodic alteration of the sign of $\langle\cos \Delta \gamma\rangle_{\text {coh }}$ from stripe to stripe. The second feature is the well-structured and asymmetric intensity of diffuse scattering observed at low angles of incidence $\alpha_{i}$ and/or scattering angle $\alpha_{f}$. Both features are due to the lateral magnetization variation on a scale smaller than the coherence range. 


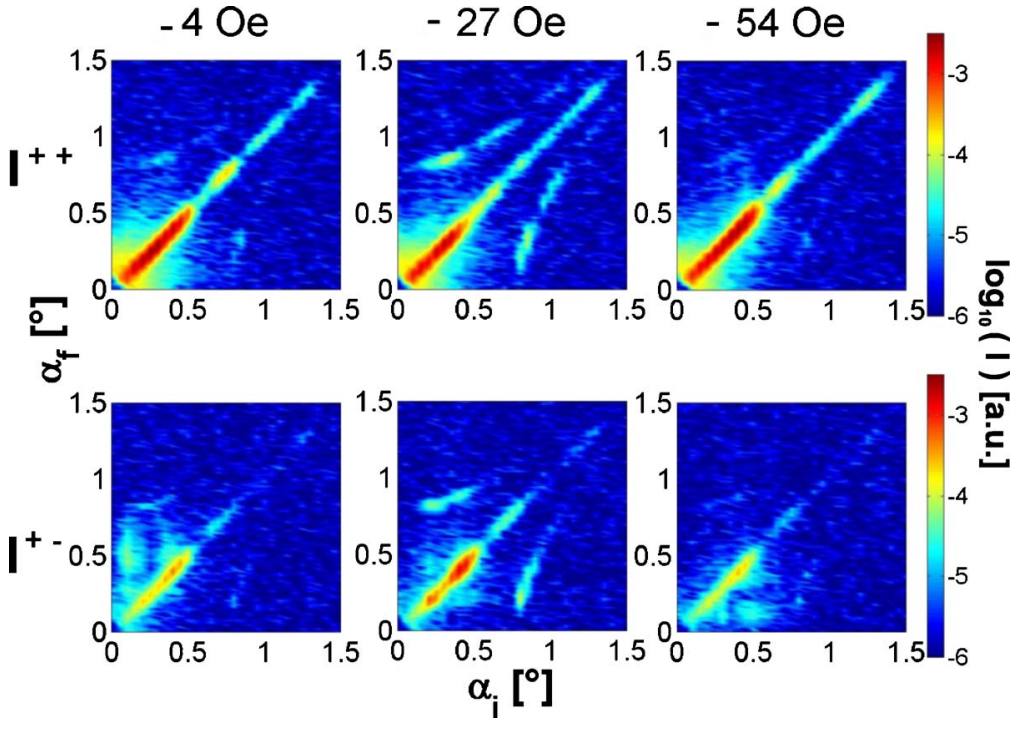

FIG. 6. (Color online) Experimental maps of the polarized neutron scattering intensity on a logarithmic scale, measured at the descending loop branch (bombarded stripes reverse first) at magnetic fields of -4 Oe (left column), -27 Oe (middle column), and -54 Oe (right column). The intensities of the $I^{++}$(top row) and $I^{+-}$(bottom row) cross-sections are plotted as a function of the angle of incidence $\alpha_{i}$, and the scattering angle $\alpha_{f}$.
The diffracted intensity reaches a maximum value in the field range where we expect antiparallel alignment of the magnetization in neighboring microstripes (second column in Fig. 6), and vanishes for parallel alignment in saturation (third column in Fig. 6). In Fig. 6, strong Bragg reflections for $n= \pm 1$ can be recognized, whereas for $n= \pm 2$ they are suppressed. This is caused by the structure factor, resulting from the equal width of adjacent microstripes in a unit cell. The direct beam shows up in the left bottom corner of the intensity maps. We find also Bragg reflections in the SF channels, which is not expected for perfect antiparallel alignment of the magnetization vector, indicative for a transverse magnetization component even in the nominal antiparallel case. This is in line with the strong specular SF reflectivity for the "antiparallel" case mentioned above. At first glance, SF diffraction indicates a periodic variation of the transverse magnetization projection perpendicular to the stripe array and to the applied field. Such variation is not favored by Maxwell's laws and would also not agree with our observations from Kerr microscopy. On the other hand, SF diffraction may easily be explained in terms of the distorted wave Born approximation (DWBA) without assumptions about the alternation of the transverse magnetization component. Moreover, DWBA is able to describe our data quantitatively by solely taking into account the alternation of longitudinal magnetization projections, if the net magnetization is tilted with respect to the field guiding neutron polarization. Within this frame, SF diffraction is a result of the superposition of two effects: Bragg diffraction due to periodical alternation of the scattering potential, and a homogeneous transverse magnetization component which mixes up neutron spin states.

More specifically, in order to apply DWBA, the magnetization distribution across the stripes (within the coherence range) is decomposed into several components as sketched in Figs. 7 and 8 for two typical magnetization arrangements in the pattern. The first component corresponds to the mean value of the magnetization (dashed arrows in the first and second panels of Figs. 7 and 8), which is homogeneous within the neutron coherence range and is tilted by the angle $\gamma$ with respect to the stripes. The second component is attrib- uted to the periodic part $\bar{\beta}=\langle\beta\rangle_{\text {coh }}$ of the tilt angle $\beta$ in individual stripes with respect to the mean magnetization direction and describes alternations of the longitudinal magnetization projection in neighboring stripes. This is illustrated in the third panels of Figs. 7 and 8. The bottom panel in Fig. 7 shows random fluctuations in the stripe magnetization due to small (ripple) domains. The second and third contributions (alternations and fluctuations) add to the difference between local and mean magnetization as illustrated in the top panel of Fig. 7.

Decomposition of magnetization as sketched in Figs. 7 and 8 serves to classify interactions responsible for particular features distinguished in Fig. 6 and to quantitatively simulate them in detail. Results of our simulations are shown in Fig. 9. The neutron interaction with the laterally homogeneous

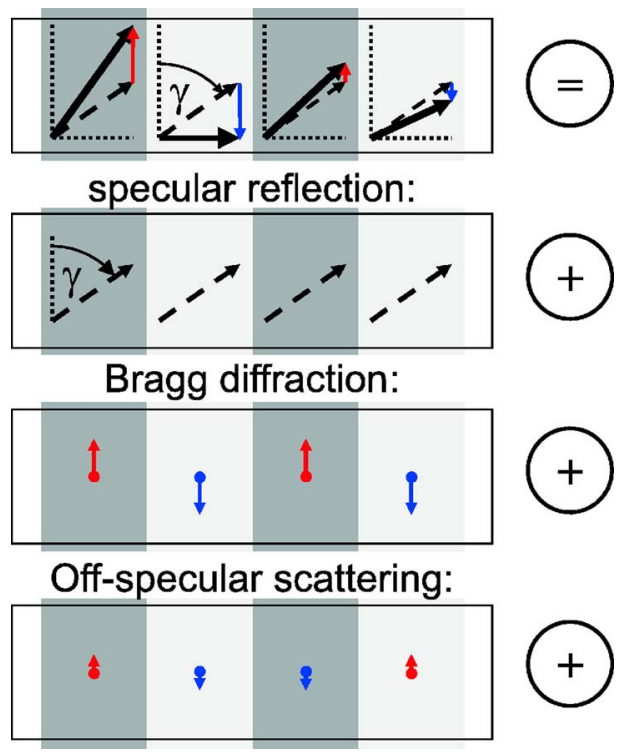

FIG. 7. (Color online) Sketch for the model used in simulations of lateral diffraction and off-specular scattering close to saturation magnetization illustrating the top view of the magnetization directions in the stripe patterns. 


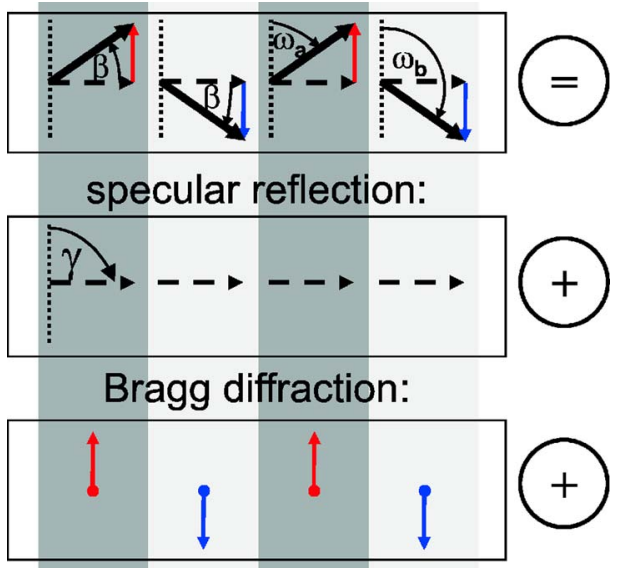

FIG. 8. (Color online) The same as in Fig. 7, but for external fields between $H_{\mathrm{c} 1}$ and $H_{\mathrm{c} 2}$.

magnetization (second panels in Figs. 7 and 8) causes optical effects. One is accounted for by SF and NSF specular reflection discussed above. Another one is the birefringence inside the mean magnetic potential which distorts spin components of the refracted neutron wave. This is in accordance with neutron spin projections onto the mean magnetization direction $^{29}$ chosen as a quantization axis inside the film. This axis may not be collinear (see second panels in Figs. 7 and 8) with the external magnetic field, which determines the quantization axis outside of the sample and guides the neutron polarization. Then inside the mean magnetic potential, neutrons fall into a mixed spin state. This means that both spin states are partially populated, and each of them can be scattered by either periodic (third panels in Figs. 7 and 8) or random (last panel in Fig. 7) parts of the interaction potential, accounted as a perturbation if scattering is small.

The neutron wave refracted into the mean optical potential and then scattered by a periodic perturbation gives rise to Bragg diffraction in DWBA (see for details Refs. 25, 29, and 31-34). Hence the observed SF diffraction is a composition of two effects: birefringent refraction into the mean optical potential and Bragg scattering of refracted neutron waves. Note that if the alternating component of magnetization is not collinear with the quantization axis parallel to the mean magnetization inside the sample, as depicted in the third panels of Figs. 7 and 8, then perturbation also mixes neutron spin states. As a result, SF Bragg diffraction may occur due to the alternation of the longitudinal magnetization in neighboring stipes, e.g., due to the change in sign of $\langle\cos \Delta \gamma\rangle_{\text {coh }}$ $=\cos \bar{\beta}$, in the presence of transverse mean magnetization. Such a situation indeed takes place in our experiment, as can be seen in the transverse Kerr sensitivity mode in the righthand image in Fig. 4.

The arguments above are also valid in the case of diffuse off-specular scattering, which is due to random fluctuations $\Delta \gamma$ around their mean values $\bar{\gamma}$ (last panel in Fig. 7), or $\Delta \beta$ around their mean values $\bar{\beta}$ (panel omitted in Fig. 8). These fluctuations not only cause diffuse scattering, but also reduce the intensity of the Bragg reflection, while the magnetic contribution to specular reflection is reduced by both regular and random deviation in angles $\beta=\bar{\beta}+\Delta \beta$. This is accounted for by the reduction of the reflection potential due to $\langle\cos \gamma\rangle_{\operatorname{coh}}$ $\leqslant 1$.

If the mean magnetization is perpendicular to the stripes (middle column in Fig. 6), we observe that diffuse scattering is suppressed, in contrast to the situation sketched in the first and the last columns, while still seen in SF channels. This is mostly due to the small longitudinal component of magnetization fluctuations (not shown in the sketch in Fig. 8 for this state). Scattering is quite symmetric and enhanced at $\alpha_{i}$ or $\alpha_{f}$ close to the values at which $\mathcal{R}^{+-}$reaches maximum values. This enhancement is due to the amplification of the neutron wave field in the optical potential, in particular, at $\alpha_{i}$ and/or $\alpha_{f}$ close to the critical angle for total reflection (Yoneda effect).

SF diffuse scattering in the first and the last columns in Fig. 6 is, on the contrary, strongly asymmetric, and its asymmetry depends on the net magnetization projection onto the field guiding neutron polarization. In the first column, offspecular scattering intensity $\mathrm{I}^{+-}$is mostly disposed at $\alpha_{f}$ $>\alpha_{i}$, while in the last one it is concentrated at $\alpha_{f}<\alpha_{i}$. In equivalence, there is also an antisymmetry between the offspecular scattering intensities in the different SF channels, $I^{+-}$and $I^{-+}$(not presented), for scattering at the same field.

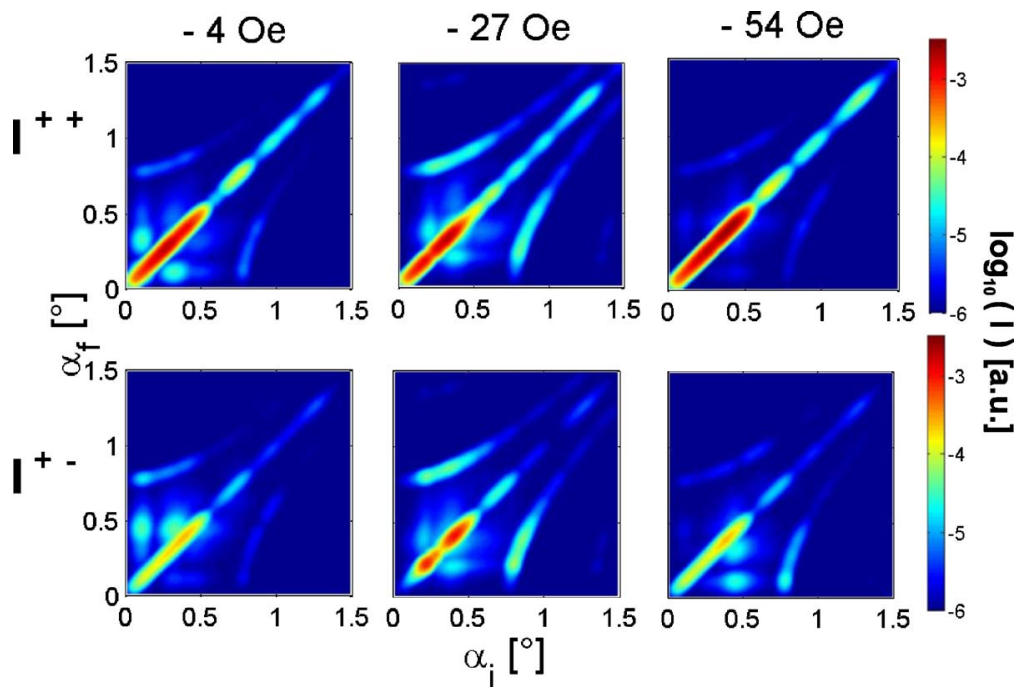

FIG. 9. (Color online) Calculated maps of the polarized neutron scattering intensity on a logarithmic scale according to the domain model discussed in the text. The calculated maps are corresponding to the experimental maps in Fig. 6 measured at the descending loop branch (bombarded stripes reverse first) at magnetic fields of -4 Oe (left column), -27 Oe (middle column), and -54 Oe (right column). The intensities of the $I^{++}$(top row) and the $I^{+-}$(bottom row) cross sections are plotted as a function of the angle of incidence $\alpha_{i}$ and the scattering angle $\alpha_{f}$. 
NSF diffuse scattering is, in contrast, symmetric. SF and NSF scattering together indicate that there are magnetization fluctuations of both longitudinal and transverse components.

Off-specular diffuse scattering is most pronounced at and just below $H_{\mathrm{c} 1}$, and at and just above $H_{\mathrm{c} 2}$. We basically account the strong off-specular diffuse SF scattering occurring around $H_{\mathrm{c} 1}$ and $H_{\mathrm{c} 2}$ for the presence of ripple domains, seen in the KM images. At fields between $H_{\mathrm{c} 1}$ and $H_{\mathrm{c} 2}$, only little diffuse SF intensity is observed, accounting for a much more regular domain state sketched in Fig. 8. In this field range, the mean magnetization orientation (dashed arrows in the first and second panels) is almost at $\gamma=90^{\circ}$.

\section{ANALYSIS AND DISCUSSION}

\section{A. Complementarity of magneto-optics and PNR}

The experimental data presented above provide rich and detailed information about the remagnetization process of laterally patterned and alternate EB stripes. The results obtained with vector-MOKE [Fig. 2(a)] and those extracted from the fits of specular PNR data (Fig. 5) are compared in Fig. 10. The results for both loop branches are presented in equivalent plots (similar to those measured via MOKE). While in MOKE measurements the magnetic field was cycled from negative to positive and again to negative fields, in PNR measurements the descending loop branch was measured after rotating the sample by $180^{\circ}$ in order to avoid negative fields because of neutron depolarization effects.

Figure 10(a) shows the field dependence of the mean projection of the normalized magnetization $\langle\cos \gamma\rangle$ onto the stripe axis. Continuous lines corresponding to the results obtained by MOKE via Eq. (1) are compared with those calculated as $\langle\cos (\bar{\gamma} \pm \Delta \gamma)\rangle=\langle\cos \bar{\gamma}\rangle\langle\cos \Delta \gamma\rangle_{\text {coh }}$ with $\langle\cos \bar{\gamma}\rangle$ and $\langle\cos \Delta \gamma\rangle_{\text {coh }}$ deduced from the fits to the PNR data. Both sets of data consistently show that the magnetization reversal passes an intermediate stage manifested by a plateau where the longitudinal projection of the mean magnetization is small and almost field independent.

This, however, does not mean that the net magnetization vanishes as to be expected for a perfect antiparallel orientation of magnetization in neighboring stripes. Instead, the set of Figs. 10(b)-10(d) shows that the magnetization vector in the plateau region is rotated into a direction almost perpendicular to the stripes, while being also reduced in length. Indeed, Figs. 10(b) and 10(d) clearly demonstrate that $|\langle\cos \gamma\rangle| \ll 1$ mostly due to $|\langle\cos \bar{\gamma}\rangle| \ll 1$, i.e., $\bar{\gamma} \approx 90^{\circ}$. At the same time, the mean magnetization proportional to $\langle\cos \Delta \gamma\rangle_{\text {coh }}$ in Fig. 10(c) is nonzero, although about half of its nominal value is lost.

Figure 10(b) shows the average over the direction of the mean magnetization vector within the coherence length as expressed by $\langle\cos \bar{\gamma}\rangle$. Because of the large laser coherence length, MOKE measurements provide the direction of the mean magnetization over the entire illuminated laser spot. In the case of PNR, an incoherent average over mean magnetization values from different coherence volumes is taken. As a fitting parameter of the specular PNR data we obtain $\langle\cos \bar{\gamma}\rangle$, which is plotted in Fig. 10(b). The curves in Fig.

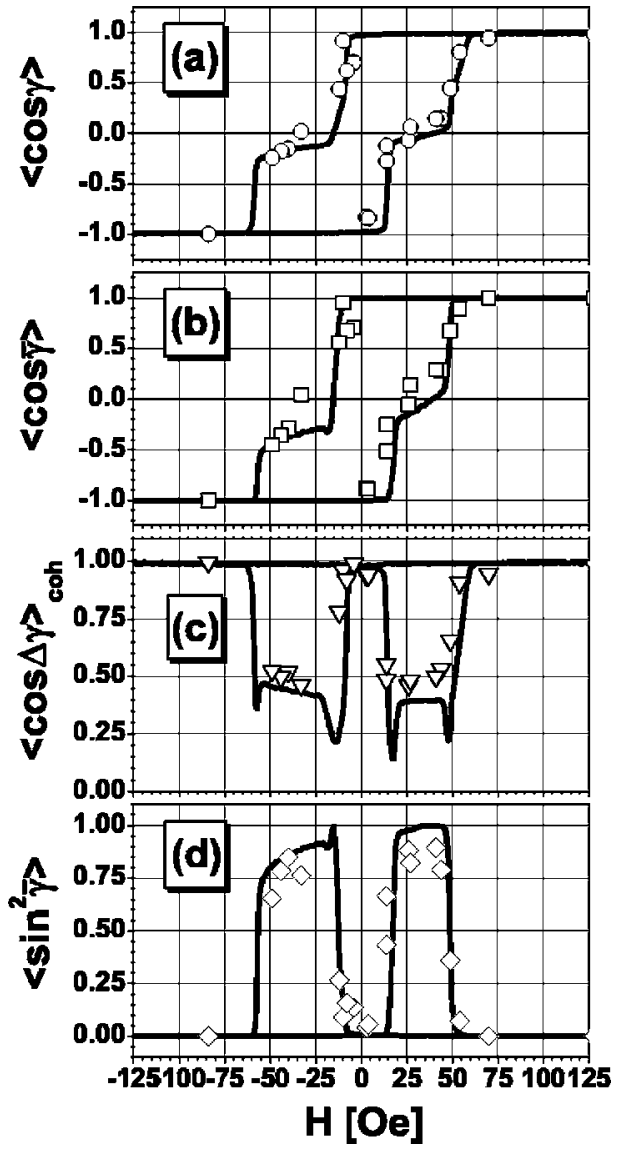

FIG. 10. Results of the fits to the PNR data (symbols) compared to vector-MOKE results (lines). (a) Field dependence of the mean projection of the normalized magnetization direction onto the stripes as determined from MOKE as $\theta_{K} / \theta_{K}^{s}$, and from the fits to the PNR data as $\langle\cos \gamma\rangle=\langle\cos \bar{\gamma}\rangle\langle\cos \Delta \gamma\rangle_{\text {coh. }}$. (b) Field dependence of the mean longitudinal normalized magnetization $\langle\cos \bar{\gamma}\rangle$ projected onto the easy axis (PNR), and determined as $\cos \left[\arctan \left(\theta_{K}^{T} / \theta_{K}^{L}\right)\right]$ from MOKE. (c) Field dependence of the mean value of magnetization $\langle\cos \Delta \gamma\rangle_{\text {coh }}(\mathrm{PNR})$, compared to $\left[\left(\theta_{K}^{L}\right)^{2}+\left(\theta_{K}^{T}\right)^{2}\right]^{1 / 2} / \theta_{K}^{s}$ determined from MOKE. (d) Field dependence of the transverse dispersion $\left\langle\sin ^{2} \bar{\gamma}\right\rangle$ projected onto the easy axis, compared to $\sin ^{2}\left[\arctan \left(\theta_{K}^{T} / \theta_{K}^{L}\right)\right]$ from MOKE.

10(b) suggest that at the coercive field $H_{\mathrm{c} 1}|\langle\cos \bar{\gamma}\rangle|$ becomes suddenly reduced. Not only the antiparallel alignment of magnetization in neighboring stripe areas leads to this reduction, but also a rotation of the magnetization out of the field direction. In the antiparallel field range, MOKE and PNR data deviate from each other. Apparently, the different coherence volumes and averaging procedures for MOKE and PNR are responsible for these differences. At $H_{\mathrm{c} 2}$, a sudden change of the direction of the mean magnetization into the direction of the field is observed.

The development of domains during the magnetization reversal can be observed in Fig. 10(c). For MOKE measurements it follows from the length of the magnetization vector $|\boldsymbol{M}| /\left|\boldsymbol{M}^{\mathrm{s}}\right|$ as determined by Eq. (2). In the fits to the specular PNR data, $\langle\cos \Delta \gamma\rangle_{\mathrm{coh}}$ is one of the fitting parameters. The general behavior is characterized by a strong reduction of the length of the magnetization vector around $H_{\mathrm{c} 1}$, a constant 
value in the antiparallel state, and an increase around $H_{\mathrm{c} 2}$, reaching its full length at saturation magnetization slightly above $H_{\mathrm{c} 2}$. In some details, MOKE and PNR provide different results. First, the values in the antiparallel state deviate from each other. Whereas from fits to the PNR data we find $\langle\cos \Delta \gamma\rangle_{\text {coh }}^{A P} \approx 0.5$, MOKE provides $|\boldsymbol{M}| /\left|\boldsymbol{M}^{\mathrm{s}}\right|^{A P} \approx 0.4$ for the ascending and $\approx 0.42-0.45$ for the descending loop branch. Second, the MOKE data show a strong decrease of $|\boldsymbol{M}| /\left|\boldsymbol{M}^{\mathrm{s}}\right|$ at both coercive fields, which is due to domains formation during the reversals from the parallel to the antiparallel $\left(H_{\mathrm{c} 1}\right)$ and from the antiparallel to the parallel $\left(H_{\mathrm{c} 2}\right)$ states. Although the MOKE data are more complete than the PNR data around the coercive fields, the PNR data for $\langle\cos \Delta \gamma\rangle_{\text {coh }}$ do not show a similar decrease of $|\boldsymbol{M}| /\left|\boldsymbol{M}^{\mathrm{s}}\right|$ at both coercive fields, as observed in the MOKE measurements. We again explain this difference by the different averaging procedures. If a stripe is partly reversed, the MOKE signal from the stripe becomes strongly reduced, while in PNR measurements each mean value of $\langle\cos \Delta \gamma\rangle$ within all coherency volumes is only slightly affected before averaging incoherently.

Even more dramatic is the averaging effect of $\sin ^{2} \bar{\gamma}$ in Fig. 10(d). While no difference exists between $\left\langle\sin ^{2} \bar{\gamma}\right\rangle$ and $\langle\sin \bar{\gamma}\rangle^{2}$ in MOKE, in PNR the difference may be quite appreciable because for $\left\langle\sin ^{2} \bar{\gamma}\right\rangle$ negative and positive magnetization components do not cancel out as it is the case for $\langle\sin \bar{\gamma}\rangle^{2}$. First, it is quite striking that MOKE as well as PNR both show a dramatic effect in $\sin ^{2} \bar{\gamma}$ which clearly accounts for a strong transverse magnetization component. Second, in the light of the previous results it is surprising that MOKE and PNR results agree with each other here, in particular at the descending branch. Apparently, variations along the stripes which are mostly responsible for the differences between MOKE and PNR data are more pronounced in the ascending loop. The good agreement between MOKE and PNR, in particular in the antiparallel field range, comes from the fact that the variations along the stripes are small.

It should be stressed that from the MOKE and specular PNR results alone one cannot draw conclusions about the origin of the mean magnetization reduction depicted in Fig. 10 (c) and thus on a microscopic arrangement of magnetic states along the hysteresis loop. However, an answer immediately follows from fits to the Bragg diffraction and diffuse scattering data shown in Fig. 9. As mentioned above, Bragg diffraction is due to periodic deviations in the angle $\Delta \gamma$ denoted as $\bar{\beta}$ in Fig. 8, while diffuse scattering is caused by random fluctuations $\Delta \beta$. The balance between Bragg and diffuse scattering intensities recorded experimentally can be reproduced via a variation of these two parameters at $\langle\cos \Delta \gamma\rangle_{\text {coh }}$ fixed at the value found from the reflectivity fit. This procedure provides a reasonably good description of the intensity maps in Fig. 6 with a value $\bar{\beta} \approx 8^{\circ}$ at $H=4$ Oe and $H=54$ Oe (left and right panels in Fig. 9), while $\bar{\beta} \approx 60^{\circ}$ at $H=27$ Oe (middle panel).

\section{B. Exchange instability of patterned EB stripes}

The magnetization reversal of the EB pattern together with the evolution of different field dependent parameters reproduced in Fig. 10 (and those providing Fig. 9) could, in principle, be modeled by micromagnetic simulations. ${ }^{35}$ Instead of performing this type of simulation, we will explain the main findings of our experiments with a simple phenomenological model. First we assume that after proper field cooling and ion-beam treatment discussed above a laterally periodic system of stripe domains in the antiferromagnetic sublayer is produced with its main axis parallel to the long axis of the stripes. The spins in the antiferromagnetic underlayer $s_{i}$ are supposed to be pinned to the AFM anisotropy field collinear with the stripes. Hence the interfacial FM spins $S_{i}$ experience the AFM induced interfacial exchange field $\boldsymbol{H}_{i}^{\mathrm{eb}}$ which is determined by the interfacial exchange energy

$$
\sum_{i} \boldsymbol{\mu}_{i} \boldsymbol{H}_{i}^{\mathrm{eb}}=-\frac{1}{2} \sum_{i}\left\langle\boldsymbol{S}_{i}\right\rangle \sum_{j} J_{i j}^{\mathrm{eb}}\left\langle\boldsymbol{s}_{j}\right\rangle,
$$

where $\left\langle\boldsymbol{S}_{i}\right\rangle$ and $\left\langle\boldsymbol{s}_{j}\right\rangle$ are mean values of FM and AFM spins, respectively, averaged over thermal fluctuations, $\boldsymbol{\mu}_{i}=\mu_{i}\left\langle\boldsymbol{S}_{i}\right\rangle$ are the magnetic moments of FM ions, and $J_{i j}^{\mathrm{eb}}$ are exchange integrals between FM and AFM spin counterparts on sites $i$ and $j$ across the FM/AFM interface.

At this point we are not interested in the microscopic mechanisms of thermo- and radio-magnetic treatment (see for details Ref. 19), which results in the creation of AFM domains of the type $a$ and $b$ corresponding to nonbombarded and bombarded areas of the sample. For our further phenomenology the only important fact is that both thermo- and radio-magnetic treatment allow one to set interfacial AFM spins in preferential directions determined by either the cooling field or the field applied at ion bombardment. This sets the effective surface exchange fields to

$$
\begin{aligned}
& \boldsymbol{\mu}_{i a} \boldsymbol{H}_{i a}^{\mathrm{eb}}=-\frac{1}{2}\left\langle\boldsymbol{S}_{i}\right\rangle \sum_{j \in a} J_{i j}^{\mathrm{eb}}\left\langle\boldsymbol{s}_{j}\right\rangle, \\
& \boldsymbol{\mu}_{i b} \boldsymbol{H}_{i b}^{\mathrm{eb}}=-\frac{1}{2}\left\langle\boldsymbol{S}_{i}\right\rangle \sum_{j \in b} J_{i j}^{\mathrm{eb}}\left\langle\boldsymbol{s}_{j}\right\rangle .
\end{aligned}
$$

Hence the interfacial exchange field $\boldsymbol{H}_{i}^{\mathrm{eb}}$ produced at site $i$ of the FM layer by frozen-in AFM spins depends on whether this site belongs to the bombarded lateral part $a$ or to the nonbombarded part $b$ of the bilayer.

If, for instance, one assumes that the FM/AFM interface is perfectly smooth and that the top monolayer of the AFM film has an uncompensated spin structure, then after thermoand radio-magnetic treatment the top AFM monolayer consists of lateral stripes of types $a$ (nonbombarded) and $b$ (bombarded) with spins frozen-in in alternative directions as sketched in Fig. 11. In reality, due to possible disorder, e.g., produced by interfacial roughness or the polycrystalline grain structure, local fields $\boldsymbol{H}_{i a}^{\mathrm{eb}}$ and $\boldsymbol{H}_{i b}^{\mathrm{eb}}$ in both regions vary from site to site. This effectively reduces the mean values $\boldsymbol{H}_{a, b}^{\mathrm{eb}}=\left\langle\boldsymbol{H}_{a, b}^{\mathrm{eb}}\right\rangle$ averaged over disorder. For the sake of simplicity, let us assume that bombardment neither affects the exchange integrals $J_{i j}^{\mathrm{eb}}=J^{\mathrm{eb}}$, nor atomic magnetic moments $\boldsymbol{\mu}_{i}$ $=\boldsymbol{\mu}$ and $\boldsymbol{s}_{j}=\boldsymbol{s}$. Then the mean interfacial effective field may accept only two values: $\boldsymbol{H}_{i a}^{\mathrm{eb}}=\boldsymbol{H}_{a}^{\mathrm{eb}}$ for one set of stripes and $\boldsymbol{H}_{i b}^{\mathrm{eb}}=-\boldsymbol{H}_{b}^{\mathrm{eb}}$ for the other set, and this dictates antiparallel 


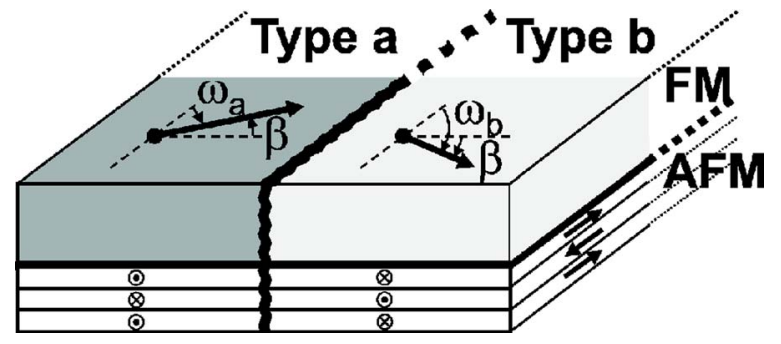

FIG. 11. (Color online) Sketch for the model of FM/AFM bilayer with two alternative directions of the EB field. A domain wall running between AFM and FM domains across AFM (bottom) and FM (top) films is sketched. Arrows indicate magnetization directions within the FM film and underlying AFM atomic layers. FM stripes of type $a$ or $b$ on top of the AFM layer belong to either bombarded or nonbombarded lateral parts, respectively.

alignment of magnetic moments in neighboring stripes.

On the other hand, spins in the FM layer are coupled to each other via the exchange interaction

$$
-\frac{1}{2} \sum_{i i^{\prime}} J_{i i^{\prime}}\left\langle\boldsymbol{S}_{i}\right\rangle\left\langle\boldsymbol{S}_{i^{\prime}}\right\rangle,
$$

which tends to homogeneously align the FM spins throughout the FM film. Therefore the FM bulk and FM/AFM interfacial interactions strongly compete, and a homogeneous magnetic state of stripes may not be possible. For thin FM layers a homogeneous FM state magnetized either in the direction of $\boldsymbol{H}_{a}^{\mathrm{eb}}$ or $\boldsymbol{H}_{b}^{\mathrm{eb}}$ may be reached only far away from the borders between bombarded and nonbombarded stripes, while in their vicinity a rapid directional change of the magnetization may prevail forming transverse domain walls. It is important to stress that a microscopic reason for such walls is rather unconventional and consists in a competition between interfacial and bulk exchange coupling.

This is in contrast to the usual role of domains which serve to close the magnetic flux inside a ferromagnet and to diminish the dipolar stray field energy around the sample ${ }^{36}$ in this way, as well as to cure a result of competition between exchange coupling and anisotropy energy. This leads to a formation of, e.g., the Landau domain pattern. The creation of domain walls costs extra energy $E_{w} \sim \sqrt{D K}$, which in uniaxial ferromagnets is determined by the exchange stiffness $D$ and the anisotropy constant $K$. Hence the number of domains (or their size) in equilibrium is determined by the balance between gain in demagnetization field energy and entropy against loss in both anisotropy and exchange coupling energies due to domain walls of the thickness $w_{d}$ $\sim \sqrt{D / K}$.

EB systems are qualitatively different, and demagnetization as well as anisotropy may play a secondary role. Indeed, the EB effect itself implies that the FM/AFM bilayer carries a macroscopic magnetic moment, thus being far from equilibrium. The frozen-in magnetic field related to this moment produces a shift in the hysteresis loop position. On the other hand, in the IBMP magnetically patterned film, FM domains are expected to be induced by the alternating interfacial exchange field $\boldsymbol{H}^{\mathrm{eb}}$, and the lateral size of domains in one direction is fixed by the width $d=d_{a}=d_{b}$ of the stripes. The thickness of the domain wall is mainly scaled with that between AFM domains, while it may also depend on the interplay between interfacial FM/AFM and bulk FM exchange energies. For the following consideration it is important that in accordance with KM observations (see Fig. 3) the thickness of the domain wall between the stripes is small so that $w \ll d$, and in first approximation can be neglected. Then the essential part of the free energy functional is approximated as

$$
\begin{aligned}
E(\gamma, \beta)= & -\frac{1}{2} J_{a b} \cos (2 \beta)-K\left(\cos ^{2} \omega_{a}+\cos ^{2} \omega_{b}\right) \\
& -\mu H_{a}^{\mathrm{eb}} \cos \omega_{a}-\mu H_{b}^{\mathrm{eb}} \cos \omega_{b},
\end{aligned}
$$

where $2 \beta=\omega_{b}-\omega_{a}$ is the angle between the magnetization vectors in neighboring stripes (see Figs. 8 and 11), $\omega_{a, b}$ and $\gamma=\left(\omega_{a}+\omega_{b}\right) / 2$ account for a possible tilt angle in the sublattice and of the net magnetization against the stripe axis, $J_{a b}$ is the exchange energy between FM spins across the plane separating FM stripe domains, and $K$ is the anisotropy constant. Here we emphasize that the anisotropy term cannot be neglected even in the case of very soft FM materials. Indeed, the EB effect is usually accompanied by a significantly increased uniaxial magnetic anisotropy, manifested in strong hysteresis phenomena with a coercive field of the same order of magnitude as (or greater than) the hysteresis loop shift. This is strong evidence in favor of that both effects have the same origin, i.e., interfacial exchange coupling. In Eq. (8), the increased anisotropy is taken into account via the phenomenologically introduced anisotropy constant $K$.

For the stripes equal in width one sets in zero external field $H_{a}^{\mathrm{eb}}=-H_{b}^{\mathrm{eb}}=H_{\mathrm{eb}}$. An external magnetic field applied along the stripe axis acts similar to the asymmetry between frozen-in exchange fields $H_{a}^{\mathrm{eb}} \neq-H_{b}^{\mathrm{eb}}$. Indeed, in the case of a field $H$ applied to the symmetric system, one should set $H_{a}^{\mathrm{eb}}=H+H_{\mathrm{eb}}$, and $H_{b}^{\mathrm{eb}}=H-H_{\mathrm{eb}}$.

At $H=0$, due to symmetry reasons, three following configurations can be realized: the "antiferromagnetic" one with zero net magnetization and alternating stripe magnetization, "ferromagnetic" order with the net magnetization directed along the stripe axis, or a mixed arrangement in which antiferromagnetic order coexists with ferromagnetic order with the net magnetization perpendicular to the stripes. The latter case is indicated in Fig. 8.

From Eq. (8) it follows that the tilt angles of the sublattice magnetization are related via the equation:

$$
\left(\mu H_{a}+K \cos \omega_{a}\right) \sin \omega_{a}=\left(\mu H_{b}+K \cos \omega_{b}\right) \sin \omega_{b} .
$$

One of the trivial solutions of this equation is $\beta= \pm \pi / 2$, i.e., $\omega_{a}=0$ and $\omega_{b}=\pi$ (or $\omega_{a}=\pi$ and $\omega_{b}=0$ ), and corresponds to the first case listed above. It can be realized within the field range $-H_{c}<H<H_{c}$, where

$$
H_{c}=\sqrt{\left(H_{\mathrm{eb}}+2 K\right)\left(H_{\mathrm{eb}}+2 K-J_{a b}\right)} .
$$

From this equation it immediately follows that pure antiferromagnetic order is only possible if 


$$
\mu H_{\mathrm{eb}}+2 K>J_{a b}
$$

i.e., at sufficiently strong EB coupling. Otherwise, a minimum of free energy at $H=0$ is reached at $\sin \beta=\mu H_{\mathrm{eb}} /\left(J_{a b}\right.$ $-2 K)$, with a net magnetic moment orthogonal to the stripe axis. This means that strong FM exchange coupling induces a coherent tilt of the lateral sublattice magnetic moments towards each other, so that a transverse FM order coexists with the AFM order along the stripe axis. The result is similar to that in so-called "weak ferromagnets," where the sublattice canting is due to spin-orbit interaction. Here, however, it has pure exchange origin.

In the symmetric case, the tilt may occur with equal probability either to the left or to the right with respect to the stripe axis. Any small ambient field misaligned with respect to the stripes can fix the net magnetization direction, if FM stripes do not fall themselves into a set of domains with magnetization vectors alternately tilted to the left and right against the stripe axis. However, this is not the case in our experiment, and the sample was always found in a state with the net magnetization tilted into the same direction practically independent of the magnetic prehistory, i.e., after cycling around the hysteresis loop. This can, in principle, be explained by the presence of a small misalignment between the stripe axis and the field cooling direction during the sample preparation described above.

Due to nonzero anisotropy, Eq. (9) is nonlinear with respect to $\sin \omega_{a, b}$ and may have either two, one stable and another unstable, or four solutions. Among them, two trivial solutions exist at $H \geqslant H_{s}$, or $H \leqslant-H_{s}$, where

$$
2 \mu H_{s}=(J+4 K)-\sqrt{J^{2}+\left(2 \mu H_{\mathrm{eb}}\right)^{2}}
$$

corresponds to the saturation field. Two other solutions may appear at either $H>H_{c}$, or at $H<-H_{c}$, where $H_{c}$ is still to be found minimizing Eq. (8) with respect to both angles $\omega_{a, b}$ for $\mu H_{\mathrm{eb}}+2 K<J_{a b}$.

In the case of four solutions, two minima may coexist at either $-H_{c}<H<-H_{s}$, or at $H_{s}<H<H_{c},{ }^{36}$ corresponding to one stable and one metastable state. At an external field variation, these minima may become equal permitting a coexistence of two types of domains comprising several stripes, e.g., one set with homogeneous magnetization collinear with the applied field and another with tilted magnetization. Further variation of the applied field may bring the system to another stable configuration of magnetic moments, i.e., into a "monodomain" state with the magnetization tilted by a certain angle with respect to the external field. The system may also be trapped in one of the states even if it becomes metastable. This particular scenario is actually observed in our experiments: Along the ascending hysteresis loop branch, the sample is maintained in the negative saturated state until it becomes absolutely unstable at $H \approx H_{s}$. Then the system rapidly enters into a monodomain state with the magnetization almost perpendicular to the field with a subsequent slow increase in magnetization and its slow rotation towards the field direction. This evolution at $H \approx H_{c}$ is interrupted by a fast flip into positive saturation via a domain mechanism of remagnetization.

In the considerations above we assumed that the stripe magnetization is independent of the applied field. However, a simple coherent rotation of magnetic moments in an external field violates the magnetic flux continuity at the interstripe borders. This condition can also readily be included in the phenomenological consideration via permitting a reduction of the mean magnetization in "unfavorable" stripes such that $\mu_{b}(H) \neq \mu_{a}(H)$, and both can vary with field. Then the flux continuity condition requires that

$$
\mu_{a} \sin \left(\gamma-\beta_{a}\right)=\mu_{b} \sin \left(\gamma+\beta_{b}\right),
$$

for $H \neq 0$ and the angles $\beta_{a} \neq \beta_{b}$. The microscopic reason for this is beyond the phenomenological approach and the field dependence of $\mu_{b}(H) \neq \mu_{a}(H)$ may be due to the ripple domains observed experimentally at $H \neq 0$ and/or because of the finite size of the boundary region between the stripes.

In conclusion, it is important to estimate conditions which allow the existence of purely antiparallel stripe magnetization orientation. Let us remind that the sum in Eq. (4) runs over sites in the vicinity of the FM/AFM interface, and therefore the $\mathrm{EB}$ exchange field contribution $\mu H^{\mathrm{eb}}$ in Eq. (8) is proportional to the squared number of interfacial ions. On the other hand, the bulk FM exchange energy across the FM domain wall is, in accordance with Eq. (7), proportional to the squared number of sites belonging to the FM domain wall running through the FM film thickness $t$. Therefore the ratio between bulk and interfacial contributions in Eq. (8) is roughly proportional to the ratio $(t / d)^{2}$. This means that one can suppress the undesirable tilt instability and overcome the constraint of Eq. (11) by either increasing the width of the stripes or reducing the FM film thickness, while keeping interfacial and bulk FM exchange couplings unchanged.

\section{SUMMARY}

We have shown that a combination of experimental techniques including magneto-optic Kerr effect, Kerr microscopy, polarized neutron reflectometry, and Bragg diffraction at grazing incidence can provide a bulk of information needed for a detailed understanding of the magnetization arrangement in exchange bias films. In the present case we have investigated a continuous ferromagnetic $\mathrm{CoFe}$ film that was exchange biased to an antiferromagnetic MnIr layer. The ferromagnetic/antiferromagnetic bilayer was subsequently modified with an ion beam such that the continuity of the ferromagnetic film was retained, but the exchange bias to the antiferromagnetic film was fixed into a stripe pattern with alternating magnetization directions. We have analyzed the magnetization reversal mechanism of this system in detail which exhibits a rich hysteresis and domain structure due to competing interfacial and intralayer exchange interactions. For a given set of parameters, including interfacial and bulk exchange coupling, stripe width, and film thickness, the intermediate state stable below the coercive field is characterized by a canted domain structure which results in a macroscopic magnetic moment directed perpendicular to the stripe direction and the exchange bias axis. A simple phenomeno- 
logical model suggests a reason for this phenomenon and gives an estimate for the range of physical parameters where one can expect a pure antiferromagnetic ordering of the stripe magnetization. This may provide directions for further advances in the design of magnetic micro- and nano-patterns with tailored properties.

\section{ACKNOWLEDGMENTS}

This study was supported by the DFG (SFB 491) and by BMBF O3ZA6BC1. One of us (B.P.T.) gratefully acknowledges a Gerhard Mercator Professorship funded through the DFG during his stay at the Ruhr-University Bochum.
*Electronic address: k.theis-broehl@rub.de; URL: http:// www.ep4.rub.de

Gerhard Mercator Guest Professor under appointment with DFG.

${ }^{1}$ Jing Shi, in Ultrathin Magnetic Structures, edited by B. Heinrich and A. C. Bland (Springer, Berlin, 2004), Vol. 4, pp. 307-331.

${ }^{2}$ S. Sun, C. B. Murray, D. Weller, L. Folks, and A. Moser, Science 287, 1989 (2000).

${ }^{3}$ A. Moser, K. Takano, D. T. Margulies, M. Albrecht, Y. Sonobe, Y. Ikeda, S. Sun, and E. E. Fullerton, J. Phys. D 35, R157 (2002).

${ }^{4}$ V. Kottler, C. Chappert, N. Essaidi, and Y. Chen, IEEE Trans. Magn. 34, 2012 (1998).

${ }^{5}$ A. Ehresmann, I. Krug, A. Kronenberger, A. Ehlers, and D. Engel, J. Magn. Magn. Mater. 280, 369 (2004).

${ }^{6}$ A. Mougin, T. Mewes, M. Jung, D. Engel, A. Ehresmann, H. Schmoranzer, J. Fassbender, and B. Hillebrands, Phys. Rev. B 63, 060409(R) (2001).

${ }^{7}$ J. Fassbender, S. Poppe, T. Mewes, A. Mougin, B. Hillebrands, D. Engel, M. Jung, A. Ehresmann, H. Schmoranzer, G. Faini, K. J. Kirk, and J. N. Chapman, Phys. Status Solidi A 189, 439447 (2002).

${ }^{8}$ C. Vieu, J. Gierak, H. Aunois, T. Aign, P. Meyer, J. P. Jamet, J. Ferré, C. Chappert, T. Devolder, V. Mathet, and H. Bernas, J. Appl. Phys. 91, 3103 (2002).

${ }^{9}$ R. Hyndman, P. Warin, J. Gierak, J. Ferré, J. N. Chapman, J. P. Jamet, V. Mathet, and C. Chappert, J. Appl. Phys. 90, 3843 (2001).

${ }^{10}$ D. McGrouther, J. N. Chapman, and F. W. M. Vanhelmont, J. Appl. Phys. 95, 7772 (2004).

${ }^{11}$ J. McCord, T. Gemming, L. Schultz, J. Fassbender, M. O. Liedke, M. Frommberger, and E. Quandt, Appl. Phys. Lett. 86, 162505 (2005).

${ }^{12}$ T. G. Kim, Y. H. Shin, J. H. Songa, M. C. Sung, I. S. Kim, D. G. You, J. Lee, K. Jeong, G. Y. Jeon, and C. N. Whang, Appl. Phys. Lett. 81, 4017 (2002).

${ }^{13}$ A. Ehresmann, Recent Res. Dev. Phys. 7, 401 (2004).

${ }^{14}$ A. Ehresmann, D. Junk, D. Engel, A. Paetzold, and K. Röll, J. Phys. D 38, 801 (2005).

${ }^{15}$ K. Theis-Bröhl, B. P. Toperverg, V. Leiner, A. Westphalen, H. Zabel, J. McCord, K. Rott, and H. Brückl, Phys. Rev. B 71, 020403(R) (2005).

${ }^{16}$ M. Kläui, C. A. F. Vaz, L. Lopez-Diaz, and J. A. C. Bland, J. Phys.: Condens. Matter 15, R985 (2003).

${ }^{17}$ R. P. Cowburn, D. K. Koltsov, A. O. Adeyeye, M. E. Well, and D. M. Tricker,Phys. Rev. Lett. 83, 1042 (1999).

${ }^{18}$ O. Fruchart, J. P. Nozières, W. Wernsdorfer, D. Givord, F. Rousseaux, and D. Decanini, Phys. Rev. Lett. 82, 1305 (1999).

${ }^{19}$ J. Schmalhorst, M. Sacher, V. Höink, G. Reiss, D. Engel, and A.
Ehresmann, Phys. Rev. B 70, 184403 (2004).

${ }^{20}$ T. Schmitte, K. Theis-Bröhl, V. Leiner, H. Zabel, S. Kirsch, and A. Carl, J. Phys.: Condens. Matter 14, 7525 (2002).

${ }^{21}$ A. Hubert and R. Schäfer, Magnetic Domains (Springer, Heidelberg, 1998).

${ }^{22}$ J. McCord, R. Schäfer, K. Theis-Bröhl, H. Zabel, J. Schmalhorst, V. Höink, H. Brückl, T. Weiss, D. Engel, and A. Ehresmann, J. Appl. Phys. 97, 10K102 (2005).

${ }^{23}$ K. Theis-Bröhl, T. Schmitte, V. Leiner, H. Zabel, K. Rott, H. Brückl, and J. McCord, Phys. Rev. B 67, 184415 (2003).

${ }^{24}$ A. Schreyer, R. Siebrecht, U. Englisch, U. Pietsch, and H. Zabel, Physica B 248, 349 (1998).

${ }^{25}$ B. P. Toperverg, A. Rühm, W. Donner, and H. Dosch, Physica B 267-268, 198 (1999).

${ }^{26}$ A. Rühm, B. P. Toperverg, and H. Dosch, Phys. Rev. B 60, 16073 (1999).

${ }^{27}$ V. Deriglazov et al. (to be published).

${ }^{28}$ J. McCord, R. Schäfer, R. Mattheis, and K.-U. Barholz, J. Appl. Phys. 93, 5491 (2003).

${ }^{29}$ B. P. Toperverg, in Polarized Neutron Scattering, Series Matter and Materials, edited by Th. Brückel and W. Schweika (Schriften des Forschungszentrum, Jülich, 2002), Vol. 12, p. 249.

${ }^{30}$ L. G. Parratt, Phys. Rev. 95, 359 (1954).

${ }^{31}$ B. P. Toperverg, K. Theis-Bröhl, and H. Zabel (to be published).

${ }^{32}$ B. P. Toperverg, Physica B 297, 160 (2001).

${ }^{33}$ B. P. Toperverg, Appl. Phys. A: Mater. Sci. Process. 74, S1560 (2002).

${ }^{34}$ B. P. Toperverg, G. P. Felcher, V. V. Metlushko, V. Leiner, R. Siebrecht, and O. Nikonov, Physica B 283, 149 (2000).

${ }^{35}$ M. J. Donahue and D. G. Porter, Interagency Report NISTIR 6376 (Sept. 1999), National Institute of Standards and Technology, Gaithersburg, MD.

${ }^{36}$ L. D. Landau and E. M. Lifshits, Electrodynamics of Continuous Media (Pergamon Press, Oxford, 1977).

${ }^{37}$ Note that the proportionality coefficient is a strongly nonlinear function of the mean magnetization modulus, which is in general unknown and is to be determined from the fit of reflectivity data to the model.

${ }^{38}$ It is worth reminding that a real domain wall serves to condense an excess of exchange energy, which is due to the tilt angle between neighboring spins, into narrow flat regions. This allows one to one gain homogeneous magnetization over much larger areas of domain bodies. In our case the interstripe borders do not have an intrinsic thermodynamical reason and just decorate the imprinted boundaries between regions with alternatively directed frozen-in EB fields. 